\title{
Acoustic Scene Analysis Based on Hierarchical Generative Model of Acoustic Event Sequence
}

\author{
Keisuke IMOTO $^{\dagger \text { a) }}$, Nonmember and Suehiro SHIMAUCHI ${ }^{\dagger b)}$, Member
}

\begin{abstract}
SUMMARY We propose a novel method for estimating acoustic scenes such as user activities, e.g., "cooking," "vacuuming," "watching TV," or situations, e.g., "being on the bus," "being in a park," "meeting," utilizing the information of acoustic events. There are some methods for estimating acoustic scenes that associate a combination of acoustic events with an acoustic scene. However, the existing methods cannot adequately express acoustic scenes, e.g., "cooking," that have more than one subordinate category, e.g., "frying ingredients" or "plating food," because they directly associate acoustic events with acoustic scenes. In this paper, we propose an acoustic scene estimation method based on a hierarchical probabilistic generative model of an acoustic event sequence taking into account the relation among acoustic scenes, their subordinate categories, and acoustic event sequences. In the proposed model, each acoustic scene is represented as a probability distribution over their unsupervised subordinate categories, called "acoustic sub-topics," and each acoustic sub-topic is represented as a probability distribution over acoustic events. Acoustic scene estimation experiments with real-life sounds showed that the proposed method could correctly extract subordinate categories of acoustic scenes.

key words: acoustic signal processing, acoustic event detection, acoustic scene analysis, bayesian inference
\end{abstract}

\section{Introduction}

Much interest has been expressed recently in applications such as monitoring elderly people[1], security surveillance [2], automatic classification of user activities and contexts [3], [4], and multimedia retrieval [5], which utilizes the information that can be obtained by analyzing various acoustic signals. There are some significant techniques for these applications; acoustic event detection (AED) analyzes various types of sounds, e.g., "footsteps," "running water," "music," "voice," to detect specific types of sounds [6], [7], and acoustic scene analysis (ASA) analyzes acoustic scenes such as user activities, e.g., "cooking," "vacuuming," "watching TV," or situations, e.g., "being on the bus," "being in a park," "meeting," on the basis of the information of acoustic events [8]. In this paper, we focus on automatic estimation of acoustic scenes, which enables automatic life-logging, the monitoring of elderly people, or multimedia retrieval.

One of the most fundamental approaches for analyzing acoustic scenes is focusing on a single acoustic event that obviously characterizes a certain acoustic scene and detect-

Manuscript received January 29, 2016.

Manuscript revised May 10, 2016.

Manuscript publicized July 19, 2016.

${ }^{\dagger}$ The authors are with NTT Media Intelligence Laboratories, NTT Corporation, Musashino-shi, 180-8585 Japan.

a) E-mail: keisuke.imoto@ieee.org

b) E-mail: shimauchi.suehiro@lab.ntt.co.jp

DOI: $10.1587 /$ transinf.2016SLP0004 ing this noticeable acoustic event. However, many acoustic scenes are characterized not by a single acoustic event but by a combination of multiple acoustic events. For example, an acoustic scene, "cooking," is marked by a combination of acoustic events including "running water," "cutting with a knife," and "heating a skillet." On the basis of this consideration, Ahaikh et al. [9] proposed a supervised user activity estimation method using a combination of multiple acoustic events. Heittola et al. [10] also proposed a content recognition method using a combination of multiple acoustic events.

An alternative strategy for acoustic scene analysis is focusing on a model of generating acoustic events from acoustic scenes. With a simple supervised generative model of acoustic events, it can be assumed that each acoustic scene has a probability distribution of acoustic events directly, and each acoustic event is generated from its distribution. We call this model the "acoustic scene model (ASM)." Kim et al. [11], Lee et al. [12], and Imoto et al. [13], [14] proposed another unsupervised generative model for analyzing acoustic scenes on the basis of the "acoustic topic model (ATM)." The ATM is an unsupervised generative model that generates acoustic event sequences from acoustic scenes through the latent structure of the acoustic scene lying in a combination of acoustic events, which is called the "acoustic topic." Specifically, the ATM characterizes acoustic scenes as a distribution of acoustic topics without utilizing the information of acoustic scenes explicitly, and this enables unsupervised analysis of acoustic scenes.

We point out here that some acoustic scenes have more than one subordinate category of an acoustic scene as shown in Fig. 1, and therefore, directly characterizing acoustic scenes with combinations of multiple acoustic events such as with the ASM or ATM is not always as expressive in modeling and estimating these acoustic scenes. For example, we consider that the acoustic scene "cooking" is marked by its subordinate categories including "preparation of ingredients," "frying ingredients," and "plating food," and each of the subordinate categories is characterized by a combination of acoustic events such as "opening a fridge," "running water," and "cutting with a knife."

In this paper, we propose an acoustic scene estimation method for an acoustic scene that has more than one subordinate category of acoustic scenes. In the proposed method, we model the generative process of acoustic event sequences as a hierarchical structure assuming that an acoustic scene is represented as a probabilistic distribution of their sub- 


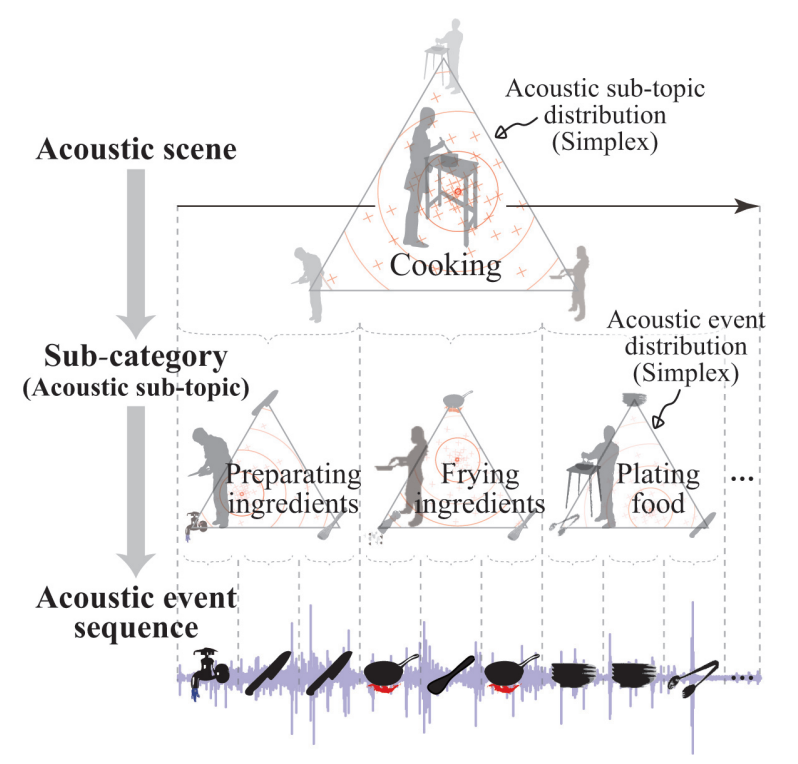

Fig. 1 Relation between acoustic scenes, acoustic sub-topics, and acoustic event sequences

ordinate categories, and each subordinate category of the acoustic scene is described as the probabilistic distribution of acoustic events. We call this model the "acoustic scene and sub-topic model (ASTM)."

The rest of this paper is as follows. In Sect. 2, we introduce the conventional probabilistic generative models of acoustic event sequences for acoustic scene analysis. In Sect. 3, we describe our proposed probabilistic generative model of an acoustic event sequence with acoustic scenes and their subordinate categories. In Sect. 4, we present an acoustic scene estimation method that includes acoustic event estimation and the parameter estimation method of our proposed model. In Sect. 5, we discuss the results of the experiments, and Sect. 6 concludes this paper.

\section{Conventional Generative Model of Acoustic Event Sequence}

In this section, we discuss two models for generating acoustic event sequences for associating acoustic scenes and combinations of acoustic events. Here, definitions of variables used in this paper are listed in Table 1.

\subsection{Acoustic Scene Model}

When modeling the relation between an acoustic scene and an acoustic event sequence with a single step generative process, it can be assumed that the acoustic scene has a probability distribution over the acoustic events, and each acoustic event is generated following its probability distribution. For instance, the ASM models a generative process of acoustic event sequences using acoustic scene information explicitly. In the ASM, the generative process of each acoustic event sequence $\boldsymbol{e}_{s}$ of acoustic signal index $s(=1,2, \ldots, S)$ can be described as shown in Fig. 2. In this model, possible
Table 1 Definition of variables

\begin{tabular}{|c|c|}
\hline Symbol & Definition \\
\hline$S$ & Total number of acoustic event sequences \\
\hline$A$ & Number of acoustic scene categories \\
\hline$T$ & Number of acoustic sub-topic categories \\
\hline$M$ & Number of acoustic event categories \\
\hline$N_{e_{s}}$ & $\begin{array}{l}\text { Number of acoustic events in acoustic event } \\
\text { sequence } \boldsymbol{e}_{s}\end{array}$ \\
\hline$s$ & Index of acoustic signals (acoustic event sequences) \\
\hline$a$ & Index of acoustic scenes \\
\hline$t$ & Index of acoustic sub-topics \\
\hline$m$ & Index of acoustic events \\
\hline$i$ & Order index of acoustic events in each acoustic signal \\
\hline $\mathcal{S}$ & Acoustic event sequence set \\
\hline$x$ & Latent variable for acoustic scene \\
\hline$z$ & Latent variable for acoustic sub-topic \\
\hline $\boldsymbol{e}_{s}$ & Acoustic event sequence of $s$ \\
\hline $\boldsymbol{e}_{s}^{*}$ & Acoustic event sequence for test signal \\
\hline $\boldsymbol{a}_{s}$ & Possible acoustic scene set in event sequence $s$ \\
\hline$\theta_{a}$ & Acoustic sub-topic distribution over acoustic scene $a$ \\
\hline$\phi_{t}$ & Acoustic event distribution over acoustic sub-topic $t$ \\
\hline$\alpha, \beta$ & Hyperparameter of Dirichlet distribution \\
\hline$\gamma_{\text {smat }}$ & $\begin{array}{l}\text { Variational parameter for multinomial distribution in } \\
\text { acoustic signal } s \text { and event } m \text { assigned to acoustic } \\
\text { scene } a \text { and sub-topic } t\end{array}$ \\
\hline Uni & Uniform distribution* \\
\hline $\operatorname{Dir}(\cdot)$ & Dirichlet distribution* \\
\hline $\operatorname{Mult}(\cdot)$ & Multinomial distribution* \\
\hline$\Gamma(\cdot)$ & Gamma function* \\
\hline
\end{tabular}

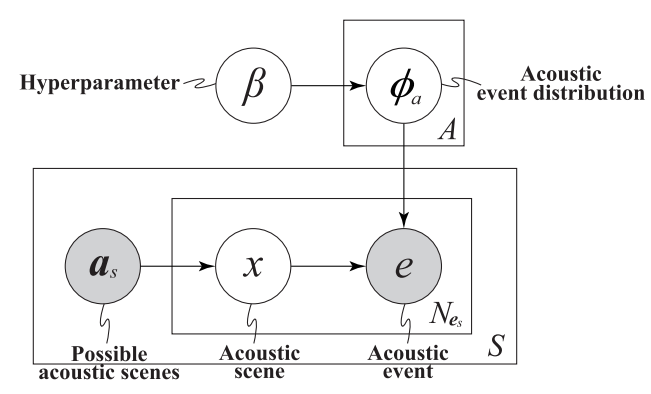

Fig. 2 Graphical model representation of acoustic scene model (ASM)

acoustic scenes in $s$ are given in advance, and one of them is generated in each time frame. Each acoustic scene has the distribution $\boldsymbol{\phi}_{x_{s i}}$ over acoustic events, and each acoustic event $e_{s i}$ is generated from this distribution. That is, acoustic scenes and events are associated through this distribution $\boldsymbol{\phi}_{x_{s i}}$. Moreover, each acoustic event distribution has a Dirichlet prior $\operatorname{Dir}(\beta)$, which is intended to avoid overfitting to a dataset. In practice, the generative process of the ASM can be expressed as follows.

Possible acoustic scenes $a_{\mathcal{S}}$ are given

Iterate $\boldsymbol{a}$ over \# of acoustic scene categories $\boldsymbol{A}$
1. Choose $\phi_{a}$ $\sim \operatorname{Dir}(\beta)$

Iterate $s$ over \# of acoustic event sequences $S$ Iterate $\boldsymbol{i}$ over \# of events in each event sequence $N_{e_{s}}$ 


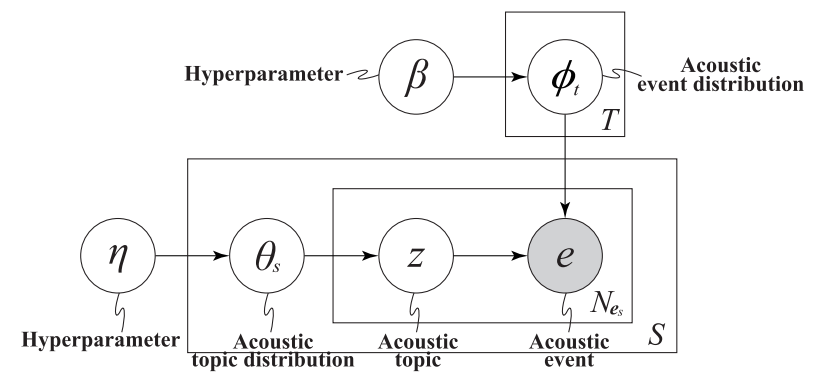

Fig. 3 Graphical model representation of acoustic topic model (ATM)

$$
\begin{array}{ll}
\text { 2. Choose } x_{s i} \mid \boldsymbol{a}_{s} & \sim \operatorname{Uni} \\
\text { 3. Choose } e_{s i} \mid \boldsymbol{\phi}_{x_{s i}}, x_{s i} & \sim \operatorname{Mult}\left(\boldsymbol{\phi}_{x_{s i}}\right) \text {, }
\end{array}
$$

To relax the temporal constraints of an acoustic event sequence and simplify the representation of the model, it is assumed that an acoustic event sequence can be described as a "bag of acoustic events (BoE)," which corresponds to the "bag of words" representation in natural language processing [16]. BoE representation assumes that each acoustic event in a sequence is exchangeable; that is, it can be hypothesized that there is no strong temporal relation between acoustic events in the short term but there is one in the long term.

\subsection{Acoustic Topic Model}

Acoustic scene estimation methods that focus on the latent structure of an acoustic scene lying in acoustic event sequences have also been proposed, and these are called the "acoustic topic model (ATM)" [11]-[13]. In the ATM, acoustic scenes are represented as a distribution of latent variables called "acoustic topics," and a generative process of acoustic event sequences $\boldsymbol{e}_{s}$ is modeled not by using acoustic scenes directly but through distributions of acoustic topics as shown in Fig. 3. In the ATM, each acoustic event sequence $\boldsymbol{e}_{s}$ has a distribution $\boldsymbol{\theta}_{s}$ of acoustic topics, and each acoustic topic $z_{s i}$ is generated from this distribution. Each topic also has the distribution $\phi_{z_{s i}}$ over acoustic events, and each acoustic event $e_{s i}$ is then generated from this distribution. That is, in this model, acoustic scenes and events are associated through the distributions $\boldsymbol{\theta}_{s}$ and $\boldsymbol{\phi}_{z_{s i}}$. Moreover, each acoustic topic distribution and event distribution has a Dirichlet prior $\operatorname{Dir}(\eta)$ and $\operatorname{Dir}(\beta)$, which are also intended to avoid overfitting to a dataset. For instance, the generative process of the ATM can be represented as follows.

\section{Iterate $\boldsymbol{t}$ over \# of acoustic topic categories $\boldsymbol{T}$}

$$
\text { 1. Choose } \phi_{t} \sim \operatorname{Dir}(\beta)
$$

Iterate $s$ over \# of acoustic event sequences $S$

2. Choose $\boldsymbol{\theta}_{s} \sim \operatorname{Dir}(\eta)$

Iterate $i$ over \# of events in each event sequence $\boldsymbol{N}_{e_{s}}$

3. Choose $z_{s i} \mid \boldsymbol{\theta}_{s} \sim \operatorname{Mult}\left(\boldsymbol{\theta}_{s}\right)$

4. Choose $e_{s i} \mid \phi_{z_{s i}}, z_{s i} \sim \operatorname{Mult}\left(\phi_{z_{s i}}\right)$.
The ATM models acoustic scenes through the distribution of acoustic topics characterized by the occurrence and co-occurrence frequency of acoustic events without the category labels of acoustic scenes. Therefore, the ATM can model acoustic scenes more flexibly, and there is a possibility that it can fit the model to the subordinate categories of acoustic scenes.

To classify acoustic scenes using the ATM, Kim et al. [20] estimated the distributions of acoustic topics for each acoustic scene and then classified acoustic scenes on the basis of a multiclass SVM that utilized acoustic scene labels and the parameters of acoustic topic distributions.

\subsection{Discussion}

As discussed above, the ASM is a supervised learning model of the relation between acoustic scenes and acoustic event sequences. In comparison, the ATM is an unsupervised one for modeling the latent structure of acoustic scenes lying in acoustic event sequences.

Some acoustic scenes have more than one subordinate category as shown in Fig. 1; however, it is not easy to arrange a data set that has all subordinate category labels of acoustic scenes. Therefore, it is impractical to model these subordinate categories of acoustic scenes using the ASM. Meanwhile, the ATM can model acoustic scenes more flexibly, and it may be able to fit the model to the subordinate categories of acoustic scenes. However, it cannot utilize the information of acoustic scenes explicitly, and therefore, we cannot control the model fitting to the subordinate categories of acoustic scenes. From these characteristics, the ASM and ATM are insufficient for modeling acoustic scenes that have more than one subordinate category, and they sometimes degrade the estimation performance of acoustic scenes.

\section{Proposed Hierarchical Generative Model of Acous- tic Event Sequence}

To address the problems in conventional models, we propose a hierarchical generative model that can associate acoustic event sequences with acoustic scenes and subordinate categories of acoustic scenes as shown in Fig. 4, and we call this model the "acoustic scene and sub-topic model (ASTM)." In the ASTM, to model an acoustic scene, we apply the acoustic scene information given by acoustic scene labels, and we treat the subordinate categories of the acoustic scenes as latent variables to capture automatically underlying structures of acoustic events.

As shown in Fig. 4, in the ASTM, possible acoustic scenes in $s$ are given in advance, and one of them is assigned in each time frame. The assigned acoustic scene $x_{s i}$ has the distribution $\boldsymbol{\theta}_{a}$ of sub-topics, and each sub-topic $z_{s i}$ is then generated from this distribution. This sub-topic also has the distribution $\phi_{z_{s i}}$ over acoustic events, and each acoustic event $e_{s i}$ is generated from this distribution. As with the 
ASM or ATM, each sub-topic distribution and event distribution has a Dirichlet prior $\operatorname{Dir}(\alpha)$ and $\operatorname{Dir}(\beta)$, which are intended to avoid overfitting. In practice, the following generative process is assumed with the ASTM.

\section{Possible acoustic scenes $a_{\mathcal{S}}$ are given}

\section{Iterate $\boldsymbol{a}$ over \# of acoustic scene categories $\boldsymbol{A}$}

$$
\text { 1. Choose } \boldsymbol{\theta}_{a} \sim \operatorname{Dir}(\alpha)
$$

\section{Iterate $\boldsymbol{t}$ over \# of acoustic sub-topic categories $\boldsymbol{T}$}

$$
\text { 2. Choose } \phi_{t} \sim \operatorname{Dir}(\beta)
$$

\section{Iterate $s$ over \# of acoustic event sequences $S$}

Iterate $\boldsymbol{i}$ over \# of events in each event sequence $\boldsymbol{N}_{e_{s}}$
3. Choose $x_{s i} \mid \boldsymbol{a}_{s} \sim$ Uni
4. Choose $z_{s i} \mid \boldsymbol{\theta}_{x_{s i}}, x_{s i} \sim \operatorname{Mult}\left(\boldsymbol{\theta}_{x_{s i}}\right)$
5. Choose $e_{s i} \mid \boldsymbol{\phi}_{z_{s i}}, z_{s i} \sim \operatorname{Mult}\left(\boldsymbol{\phi}_{z_{s i}}\right)$.

Since acoustic scene labels are available for acoustic scene modeling explicitly, the ASTM has an advantage in that it can tailor the subordinate category layer modeling to fit each acoustic scene. This hierarchical modeling offers the combined benefits of the ASM and ATM to our proposed model.

The generative probability of $\boldsymbol{e}_{s}: p\left(\boldsymbol{e}_{s} \mid \alpha, \beta, \boldsymbol{a}_{s}\right)$ can be written as

$$
\begin{aligned}
p\left(\boldsymbol{e}_{s} \mid \alpha, \beta, \boldsymbol{a}_{s}\right)= & \prod_{i=1}^{N_{e_{s}}} p\left(e_{s i} \mid \alpha, \beta, \boldsymbol{a}_{s}\right) \\
= & \iint \prod_{i=1}^{N_{e_{s}}} \sum_{a=1}^{A} \sum_{t=1}^{T} \operatorname{Uni}\left(x_{s i} \mid \boldsymbol{a}_{s}\right) p\left(z_{s i} \mid x_{s i}, \boldsymbol{\theta}_{x_{s i}}\right) \operatorname{Dir}\left(\boldsymbol{\theta}_{x_{s i}} \mid \alpha\right) \\
& \cdot p\left(e_{s i} \mid z_{s i}, \boldsymbol{\phi}_{z_{s i}}\right) \operatorname{Dir}\left(\boldsymbol{\phi}_{z_{s i}} \mid \beta\right) d \boldsymbol{\phi} d \boldsymbol{\theta} \\
= & \prod_{i=1}^{N_{e_{s}}} \frac{1}{A_{s}} \sum_{a \in \boldsymbol{a}_{s}} \int \frac{\Gamma(T \alpha)}{\Gamma(\alpha)^{T}} \prod_{t=1}^{T} \theta_{a t}^{\alpha-1} \sum_{t=1}^{T} \theta_{x_{s i} i z_{s i}} \\
& \cdot \int \frac{\Gamma(M \beta)}{\Gamma(\beta)^{M}} \prod_{m=1}^{M} \phi_{t m}^{\beta-1} \cdot \phi_{z_{s i}, e_{s i}} d \boldsymbol{\phi} d \boldsymbol{\theta}
\end{aligned}
$$

where $A_{s}$ is the number of possible acoustic scenes in each

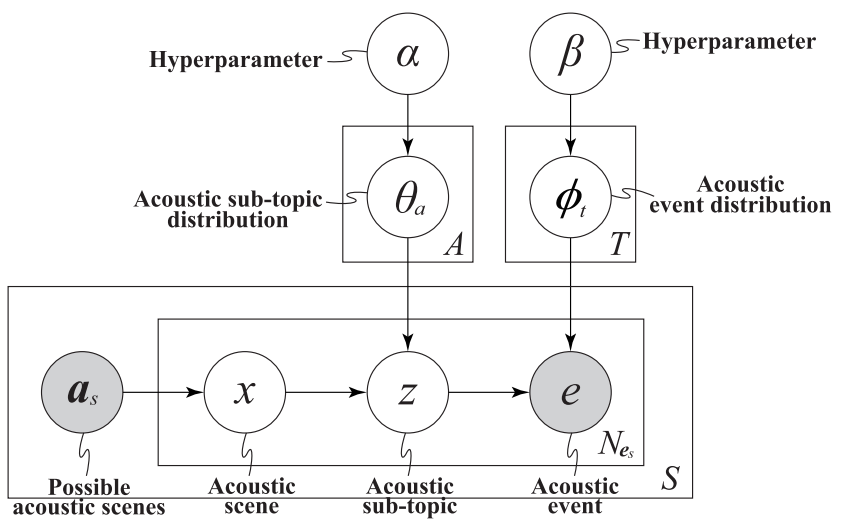

Fig. 4 Graphical model representation of proposed method (acoustic scene and sub-topic model: ASTM) acoustic event sequence, $\phi_{z_{s i}, e_{s i}}$ is the generative probability of $e_{s i}$ in $z_{s i}$, and $\theta_{x_{s i}, z_{s i}}$ is the generative probability of $z_{s i}$ in $x_{s i}$.

\section{Acoustic Scene Estimation Based on ASTM}

\subsection{Acoustic Scene Estimation Overview}

In this section, we introduce an acoustic scene estimation method with the ASTM. To estimate the acoustic scene that an acoustic event sequence $\boldsymbol{e}_{s}^{*}$ indicates with the ASTM, we need to infer an acoustic scene that maximizes the posterior probability $p\left(x_{e_{s}^{*}}=a \mid \boldsymbol{e}_{s}^{*}, \boldsymbol{\gamma}\right)$. As shown in Fig. 5, the acoustic scene estimation system has a three-step process: recognizing acoustic event sequences from acoustic signals, estimating acoustic scene model parameters, and predicting acoustic scenes with estimated model parameters. We now discuss each step as follows.

\subsection{Acoustic Feature Extraction and Event Recognition}

To estimate $\boldsymbol{e}_{s}$ or $\boldsymbol{e}_{s}^{*}$, we segment long-term input signals and extract acoustic feature vectors frame by frame. Then, we recognize acoustic events in every frame and output an $\boldsymbol{e}_{s}$ or $\boldsymbol{e}_{s}^{*}$ (composed of multiple acoustic event recognition results).

As the acoustic features, we choose Mel-frequency cepstral coefficients (MFCCs). These are well known features for speech recognition and speech synthesis, and MFCC features have also been used in AED tasks [6], in which they performed well.

Many acoustic event recognition approaches are based on the Gaussian mixture model (GMM) or hidden Markov model (HMM) [6], [7], which defines acoustic events with manually labeled data. However, it is difficult to label every possible acoustic event since there are many different types of sounds. In view of this difficulty, our proposed acoustic scene model represents acoustic scenes and subtopics as distributions over multiple acoustic sub-topics and events, respectively, so there is no need to label each acoustic event for acoustic scene modeling. Therefore, we apply the unsupervised technique of Gaussian clustering (GC) to model acoustic events. MFCC feature vectors in the learning set for acoustic event modeling are classified by

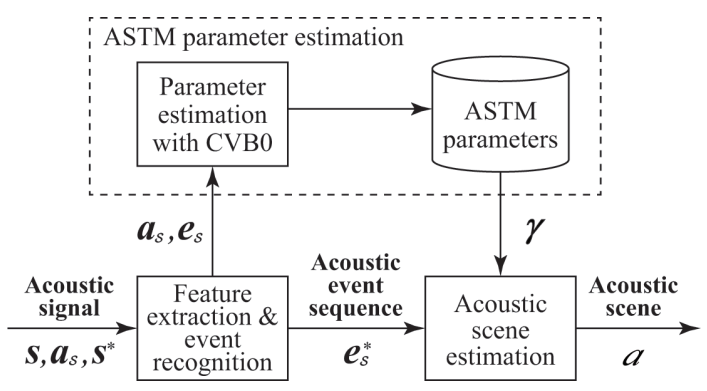

Fig. 5 Acoustic scene estimation system overview 
using GC with the expectation-maximization (EM) algorithm [17], and each Gaussian component is modeled as a single acoustic event. To recognize acoustic events, we estimate acoustic events that maximize posterior probability for the model, and the acoustic event recognition step outputs the acoustic event sequence of multiple event recognition results.

\subsection{ASTM Parameter Estimation}

The ASTM includes model parameters $\boldsymbol{x}, \boldsymbol{z}, \boldsymbol{\theta}_{a}, \boldsymbol{\phi}_{t}$ to be estimated for analyzing acoustic scenes. In this paper, we introduce a parameter estimation method that uses the collapsed variational Bayes with 0th-order approximation (CVB0) [18]. The Bayesian inference based on CVB0 shows a lower calculation cost than collapsed Gibbs sampling (CGS) and naive variational Bayes (VB) [19] and a slightly worse performance than that of CGS [20], [21]. Since we give weight to the balance between a calculation cost and parameter estimation performance, we thus apply CVB0 to estimate parameters of the ASTM.

To infer the model parameters of the ASTM, we need to estimate the posterior distribution of all unknown parameters for joint distribution. However, it is intractable to estimate a true posterior distribution $p(\boldsymbol{x}, \boldsymbol{z}, \boldsymbol{\theta}, \boldsymbol{\phi} \mid \boldsymbol{e})$. Therefore, we introduce a variational distribution $q(\boldsymbol{x}, \boldsymbol{z}, \boldsymbol{\theta}, \boldsymbol{\phi})$ and approximate it to a posterior distribution iteratively. In CVB0, we first calculate a lower bound on a marginal log likelihood $\mathcal{F}[q]$ by using Jensen's inequality.

$$
\begin{aligned}
\mathcal{L}(\boldsymbol{e}) & \equiv \log p\left(\boldsymbol{e} \mid \alpha, \beta, \boldsymbol{a}_{\mathcal{S}}\right) \\
& =\log \iint \sum_{\boldsymbol{x}} \sum_{z} q(\boldsymbol{x}, \boldsymbol{z}, \boldsymbol{\theta}, \boldsymbol{\phi}) \frac{p\left(\boldsymbol{e}, \boldsymbol{x}, \boldsymbol{z}, \boldsymbol{\theta}, \boldsymbol{\phi} \mid \alpha, \beta, \boldsymbol{a}_{\mathcal{S}}\right)}{q(\boldsymbol{x}, \boldsymbol{z}, \boldsymbol{\theta}, \boldsymbol{\phi})} d \boldsymbol{\theta} d \boldsymbol{\phi} \\
& \geq \iint \sum_{\boldsymbol{x}} \sum_{z} q(\boldsymbol{x}, \boldsymbol{z}, \boldsymbol{\theta}, \boldsymbol{\phi}) \log \frac{p\left(\boldsymbol{e}, \boldsymbol{x}, \boldsymbol{z}, \boldsymbol{\theta}, \boldsymbol{\phi} \mid \alpha, \beta, \boldsymbol{a}_{\mathcal{S}}\right)}{q(\boldsymbol{x}, \boldsymbol{z}, \boldsymbol{\theta}, \boldsymbol{\phi})} d \boldsymbol{\theta} d \boldsymbol{\phi} \\
& \equiv \mathcal{F}[q] .
\end{aligned}
$$

Here, $\mathcal{L}(\boldsymbol{e})$ and $\mathcal{F}[q]$ can be represented as the following relational expression using the KL divergence.

$$
\begin{aligned}
\mathcal{L}(\boldsymbol{e})-\mathcal{F}[q]=\log p\left(\boldsymbol{e} \mid \alpha, \beta, \boldsymbol{a}_{\mathcal{S}}\right)-\iint \sum_{\boldsymbol{x}} \sum_{z} q(\boldsymbol{x}, \boldsymbol{z}, \boldsymbol{\theta}, \boldsymbol{\phi}) \\
\cdot \log \frac{p\left(\boldsymbol{e}, \boldsymbol{x}, \boldsymbol{z}, \boldsymbol{\theta}, \boldsymbol{\phi} \mid \alpha, \beta, \boldsymbol{a}_{\mathcal{S}}\right)}{q(\boldsymbol{x}, \boldsymbol{z}, \boldsymbol{\theta}, \boldsymbol{\phi})} d \boldsymbol{\theta} d \boldsymbol{\phi} \\
=\iint \sum_{\boldsymbol{x}} \sum_{z} q(\boldsymbol{x}, \boldsymbol{z}, \boldsymbol{\theta}, \boldsymbol{\phi}) \log \frac{q(\boldsymbol{x}, \boldsymbol{z}, \boldsymbol{\theta}, \boldsymbol{\phi})}{p\left(\boldsymbol{x}, \boldsymbol{z}, \boldsymbol{\theta}, \boldsymbol{\phi} \mid \boldsymbol{e}, \alpha, \beta, \boldsymbol{a}_{\mathcal{S}}\right)} d \boldsymbol{\theta} d \boldsymbol{\phi} \\
=\mathrm{KL}\left(q(\boldsymbol{x}, \boldsymbol{z}, \boldsymbol{\theta}, \boldsymbol{\phi}) \| p\left(\boldsymbol{x}, \boldsymbol{z}, \boldsymbol{\theta}, \boldsymbol{\phi} \mid \boldsymbol{e}, \alpha, \beta, \boldsymbol{a}_{\mathcal{S}}\right)\right) .
\end{aligned}
$$

This means that we can obtain the appropriate variational parameters by maximizing the lower bound $\mathcal{F}[q]$, that is, minimizing the KL divergence. For this $\mathcal{F}[q]$, we apply the following mean field approximation.

$$
\begin{aligned}
q(\boldsymbol{x}, \boldsymbol{z}, \boldsymbol{\theta}, \boldsymbol{\phi}) & =q(\boldsymbol{\theta}, \boldsymbol{\phi} \mid \boldsymbol{x}, \boldsymbol{z}) q(\boldsymbol{x}, \boldsymbol{z}) \\
& =p\left(\boldsymbol{\theta}, \boldsymbol{\phi} \mid \boldsymbol{e}, \boldsymbol{x}, \boldsymbol{z}, \alpha, \beta, \boldsymbol{a}_{\mathcal{S}}\right) q(\boldsymbol{x}, \boldsymbol{z}) .
\end{aligned}
$$

Here, $q(\boldsymbol{\theta}, \boldsymbol{\phi} \mid \boldsymbol{x}, \boldsymbol{z})$ is defined with no restriction, and therefore, $\mathcal{F}[q]$ is maximized when $q(\boldsymbol{\theta}, \boldsymbol{\phi} \mid \boldsymbol{x}, \boldsymbol{z})=p(\boldsymbol{\theta}, \boldsymbol{\phi} \mid \boldsymbol{e}, \boldsymbol{x}, \boldsymbol{z}, \alpha$, $\beta, \boldsymbol{a}_{\mathcal{S}}$ ). By substituting Eq. (4) into Eq. (2), we obtain $\mathcal{F}[q]$ as follows.

$$
\begin{aligned}
\mathcal{F}[q]= & \iint \sum_{\boldsymbol{x}} \sum_{z} q(\boldsymbol{x}, \boldsymbol{z}, \boldsymbol{\theta}, \boldsymbol{\phi}) \\
& \cdot \log \frac{p\left(\boldsymbol{e}, \boldsymbol{x}, \boldsymbol{z} \mid \alpha, \beta, \boldsymbol{a}_{\mathcal{S}}\right) p\left(\boldsymbol{\theta}, \boldsymbol{\phi} \mid \boldsymbol{e}, \boldsymbol{x}, \boldsymbol{z}, \alpha, \beta, \boldsymbol{a}_{\mathcal{S}}\right)}{q(\boldsymbol{x}, \boldsymbol{z}, \boldsymbol{\theta}, \boldsymbol{\phi})} d \boldsymbol{\theta} d \boldsymbol{\phi} \\
= & \sum_{\boldsymbol{x}} \sum_{z} q(\boldsymbol{x}, \boldsymbol{z}) \iint q(\boldsymbol{\theta}, \boldsymbol{\phi} \mid \boldsymbol{x}, \boldsymbol{z}) \log \frac{p\left(\boldsymbol{e}, \boldsymbol{x}, \boldsymbol{z} \mid \alpha, \beta, \boldsymbol{a}_{\mathcal{S}}\right)}{q(\boldsymbol{x}, \boldsymbol{z})} d \boldsymbol{\theta} d \boldsymbol{\phi} \\
= & \sum_{\boldsymbol{x}} \sum_{z} q(\boldsymbol{x}, \boldsymbol{z}) \log p\left(\boldsymbol{e}, \boldsymbol{x}, \boldsymbol{z} \mid \alpha, \beta, \boldsymbol{a}_{\mathcal{S}}\right) \\
& -\sum_{\boldsymbol{x}} \sum_{z} q(\boldsymbol{x}, \boldsymbol{z}) \log q(\boldsymbol{x}, \boldsymbol{z}) .
\end{aligned}
$$

Considering that $p\left(\boldsymbol{x}=a \mid \boldsymbol{a}_{\mathcal{S}}\right)$ is assumed to be a uniform distribution and that the joint distribution of the Dirichlet distribution and the multinomial distribution can be represented by using the gamma function, the first term of the right side in Eq. (5) can be calculated as follows,

$$
\begin{aligned}
\sum_{x} \sum_{z} q(\boldsymbol{x}, \boldsymbol{z}) \log p\left(\boldsymbol{e}, \boldsymbol{x}, \boldsymbol{z} \mid \alpha, \beta, \boldsymbol{a}_{\mathcal{S}}\right) \\
=\sum_{\boldsymbol{x}} \sum_{z} \prod_{s} \prod_{i} q\left(x_{s i}, z_{s i}\right) \log \prod_{s} \prod_{i} \frac{1}{A_{s}} \\
\cdot \prod_{a}\left\{\frac{\Gamma(T \alpha)}{\Gamma(\alpha)^{T}} \cdot \frac{\prod_{t} \Gamma\left(\alpha+n_{. a t}\right)}{\Gamma\left(T \alpha+n_{. a .}\right)}\right\} \prod_{t}\left\{\frac{\Gamma(M \beta)}{\Gamma(\beta)^{M}} \cdot \frac{\prod_{m} \Gamma\left(\beta+n_{. m . t}\right)}{\Gamma\left(M \beta+n_{. . t}\right)}\right\},
\end{aligned}
$$

where $q\left(x_{s i}, z_{s i}\right)=\gamma_{s e_{s i} x_{s i} z_{s i}}$ and $n_{s e_{s i} a t}$ are the expected probability and the number of acoustic events in acoustic event sequence $s$ and event $e_{s i}$ assigned to acoustic scene $a$ and sub-topic $t$. The symbol "." in the subscript indicates the summation in the corresponding variable. Detailed derivations of Eqs. (6), (8), and (10) are shown in Appendix. Then, the second term of the right side in Eq. (5) can be also calculated as follows.

$$
\begin{aligned}
& \sum_{\boldsymbol{x}} \sum_{z} q(\boldsymbol{x}, \boldsymbol{z}) \log q(\boldsymbol{x}, \boldsymbol{z}) \\
& =\sum_{\boldsymbol{x}} \sum_{z} \prod_{s} \prod_{i} q\left(x_{s i}, z_{s i}\right) \log \prod_{s} \prod_{i} q\left(x_{s i}, z_{s i}\right) \\
& =\sum_{\boldsymbol{x}} \sum_{z} \prod_{s} \prod_{i} \prod_{a} \prod_{t} \gamma_{s e_{s i t} i t}^{\delta_{\text {siat }}} \sum_{s} \sum_{i} \sum_{a} \sum_{t} \log \gamma_{\text {sesiat }}^{\delta_{\text {siat }}},
\end{aligned}
$$

where $\delta_{\text {siat }}$ equals 1 when an $i$-th acoustic event in an $s$ th acoustic event sequence indicates acoustic scene $a$ and acoustic sub-topic $t$, and otherwise, the $\delta_{\text {siat }}$ equals 0 . To maximize $\mathcal{F}[q]$, we then update $q\left(x_{s i}, z_{s i}\right)$ iteratively on the basis of $\partial \mathcal{F}\left[q\left(x_{s i}, z_{s i}\right)\right] / \partial q\left(x_{s i}, z_{s i}\right)=0$. The partial derivatives of Eqs. (6) and (7) can be calculated as Eqs. (8) and (9).

$$
\begin{gathered}
\frac{\partial}{\partial \gamma_{s s_{s i} a t}} \sum_{\boldsymbol{x}} \sum_{z} q(\boldsymbol{x}, \boldsymbol{z}) \log p\left(\boldsymbol{e}, \boldsymbol{x}, z \mid \alpha, \beta, \boldsymbol{a}_{\mathcal{S}}\right) \\
=\sum_{\boldsymbol{x}^{\prime s i}, x_{s i}=a} \sum_{z^{\prime s i}, z_{s i}=t} \prod_{s^{\prime}, i^{\prime} \neq s, i} \prod_{a^{\prime}} \prod_{t^{\prime}} \gamma_{s^{\prime} e_{s^{\prime} i^{\prime}} \delta^{\prime} t^{\prime} t^{\prime}}^{s^{\prime} t^{\prime}}
\end{gathered}
$$




$$
\begin{aligned}
& \cdot \log p\left(\boldsymbol{e}, \boldsymbol{x}^{/ s i}, x_{s i}=a, \boldsymbol{z}^{/ s i}, z_{s i}=t \mid \alpha, \beta, \boldsymbol{a}_{\mathcal{S}}\right)
\end{aligned}
$$

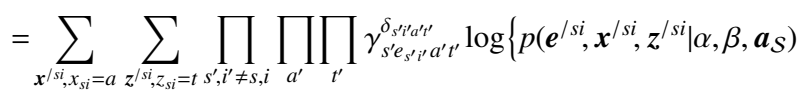

$$
\begin{aligned}
& \cdot \frac{1}{A_{s}} \frac{\alpha+n_{. . t^{\prime} t^{\prime}}^{/ s i}}{T \alpha+n_{. . a^{\prime}}^{\prime s i}}+\frac{\beta+n_{. s_{s}^{\prime} i^{\prime}, t^{\prime}}^{/ s i}}{M \beta+n_{. . . t^{\prime}}^{s s i}},
\end{aligned}
$$

where the superscript "/si" means that acoustic event $e_{s i}$ is excluded when calculating the corresponding variable.

$$
\begin{aligned}
& \frac{\partial}{\partial \gamma_{s_{s i} i t}} \sum_{\boldsymbol{x}} \sum_{z} q(\boldsymbol{x}, \boldsymbol{z}) \log q(\boldsymbol{x}, \boldsymbol{z})
\end{aligned}
$$

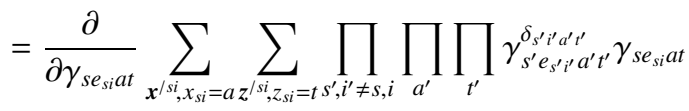

$$
\begin{aligned}
& \cdot\left\{\sum_{s^{\prime}, i^{\prime} \neq s, i} \sum_{a^{\prime}} \sum_{t^{\prime}} \log \gamma_{s^{\prime} e_{s^{\prime} i^{\prime}} a^{\prime} t^{\prime}}^{\delta_{s^{\prime}{ }^{\prime} \prime^{\prime}}}+\log \gamma_{s e_{s i} a t}\right\} \\
& \propto \log \gamma_{\text {se }_{s i} a t}+\text { const. }
\end{aligned}
$$

Substituting Eqs. (8) and (9) into $\partial \mathcal{F}\left[q\left(x_{s i}, z_{s i}\right)\right] / \partial q\left(x_{s i}, z_{s i}\right)$ $=0$ and maximizing $\mathcal{F}\left[q\left(x_{s i}, z_{s i}\right)\right]$ for $\gamma_{s e_{s i} a t}[22]$, we obtain the following update.

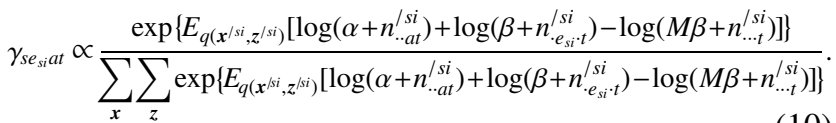

Because the computation cost of each expectation in $\gamma_{s e_{s i} a t}$ is expensive, for the ASTM, we apply the Taylor series approximation to $E_{q\left(\boldsymbol{x}^{/ s i}, \boldsymbol{z}^{/ s i}\right)}\left[\log \left(\alpha+n_{\cdot \cdot a t} / s i\right)\right]$ at $E_{q\left(\boldsymbol{x}^{/ s i}, z^{/ s i}\right)}\left[n_{\cdot \cdot a t}^{/ s i}\right]$ as follows.

$$
\begin{aligned}
& E_{q\left(x^{/ s i}, z^{/ s i}\right)}\left[\log \left(\alpha+n_{\cdot \cdot a t}^{/ s i}\right)\right] \\
& =E_{q\left(\boldsymbol{x}^{/ s i}, z^{\prime s i}\right)}\left[\log \left(\alpha+E_{q\left(\boldsymbol{x}^{\prime s i}, z^{\prime s i}\right)}\left[n_{* a t}^{/ s i}\right]\right)+\frac{E_{q\left(\boldsymbol{x}^{/ s i}, z^{/ s i}\right)}\left[n_{* a t}^{/ s i}\right]-n_{\cdot \cdot a t}^{/ s i}}{\alpha+E_{q\left(\boldsymbol{x}^{/ s i}, z^{/ s i}\right)}\left[n_{\cdot \cdot a t}^{/ s i}\right]}\right. \\
& \left.-\frac{\operatorname{Var}_{q\left(\boldsymbol{x}^{/ s i}, z^{/ s i}\right)}\left[n_{\cdot a t}^{/ s i}\right]}{2\left(\alpha+E_{q\left(\boldsymbol{x}^{/ s,}, z^{/ s i}\right)}\left[n_{* \cdot a t}^{/ s i}\right]\right)^{2}}+\cdots\right] .
\end{aligned}
$$

Furthermore, we utilize only the zeroth-order term of the Taylor series and obtain the following approximation.

$$
\begin{aligned}
E_{q\left(\boldsymbol{x}^{\prime s i}, z^{/ s i}\right)}\left[\log \left(\alpha+n_{* a t}^{\prime s i}\right)\right] & \approx E_{q\left(\boldsymbol{x}^{/ s i}, z^{/ s i}\right)}\left[\log \left(\alpha+E_{q\left(\boldsymbol{x}^{/ s i}, z^{/ s i}\right)}\left[n_{\cdot \cdot a t}^{/ s i}\right]\right)\right] \\
& =\log \left(\alpha+E_{q\left(\boldsymbol{x}^{\prime s i}, z^{/ s i}\right)}\left[n_{\cdot \cdot a t}^{/ s i}\right]\right) \\
& \approx \log \left(\alpha+\sum_{s^{\prime}, i^{\prime} \neq s, i} \gamma_{s^{\prime} e_{s^{\prime} i^{\prime}} a t}\right) \\
& \triangleq \log \left(\alpha+N_{a t}^{/ s i}\right),
\end{aligned}
$$

where we consider that $E_{q\left(x^{\prime s i}, z^{\prime s i}\right)}\left[n_{* a t}^{/ s i}\right] \approx \sum_{s^{\prime}, i^{\prime} \neq s, i} \gamma_{s^{\prime} e_{s^{\prime} i^{\prime}} a t}$ when $n_{\cdot a t}^{/ s i} \gg 0$ [18]. By applying the same approximation to $E_{q\left(\boldsymbol{x}^{/ s i}, z^{/ s i}\right)}\left[\log \left(\beta+n_{\cdot e_{s i t}}^{/ s i}\right)\right]$ and $E_{q\left(\boldsymbol{x}^{/ s i}, z^{s i}\right)}\left[\log \left(M \beta+n_{\cdots t}^{/ s i}\right)\right]$, we finally obtain the following update for ASTM.

$$
\gamma_{s e_{s i} a t} \propto\left(\alpha+N_{a t}^{/ s i}\right)\left(\beta+N_{t m}^{/ s i}\right)\left(M \beta+N_{t}^{/ s i}\right)^{-1} .
$$

To estimate appropriate parameters, this update is repeated until a convergence condition is satisfied. After this iteration, we can estimate $\theta_{a t}$ and $\phi_{t m}$ in the following equations with the resultant $N_{a t}$ and $N_{t m}$, respectively.

$$
\begin{aligned}
\theta_{a t} & =\frac{\alpha+N_{a t}}{T \alpha+\sum_{t} N_{a t}} \\
\phi_{t m} & =\frac{\beta+N_{t m}}{M \beta+\sum_{m} N_{t m}} .
\end{aligned}
$$

\subsection{Acoustic Scene Estimation}

To estimate acoustic scenes for acoustic signal $s^{*}$, for which possible acoustic scenes are unlabeled, we need to calculate $\arg \max \log p\left(a \mid \boldsymbol{e}_{s}^{*}\right)$ as follows.

$$
\begin{aligned}
\underset{a}{\arg \max } \log p\left(a \mid \boldsymbol{e}_{s}^{*}\right) & =\underset{a}{\arg \max } \log \prod_{i=1}^{N_{s}^{*}} p\left(a \mid e_{s i}^{*}\right) \\
& \approx \underset{a}{\arg \max } \sum_{i=1}^{N_{s}^{*}} \log q\left(a \mid e_{s i}^{*}\right) \\
& \propto \underset{a}{\arg \max } \sum_{i=1}^{N_{s}^{*}} \log \sum_{t=1}^{T} \gamma_{s e_{s i} a t}^{*},
\end{aligned}
$$

where $a \in a_{\mathcal{S}}$ is all possible acoustic scenes for all data sets. This is achieved by the MAP estimation of $\gamma_{s_{s i} i t}^{*}$ using the trained ASTM parameters. In practice, we maximize $q\left(\boldsymbol{x}^{*}, \boldsymbol{z}^{*}\right)$ in the following equation in a similar way described above.

$$
\begin{aligned}
\mathcal{L}\left(\boldsymbol{e}^{*}\right) \equiv & \log p\left(\boldsymbol{e}^{*} \mid \alpha, \beta, \boldsymbol{a}_{s}^{*}, \boldsymbol{e}, \boldsymbol{a}_{\mathcal{S}}\right) \\
\geq & \sum_{\boldsymbol{x}^{*}} \sum_{z^{*}} q\left(\boldsymbol{x}^{*}, \boldsymbol{z}^{*}\right) \\
& \cdot \log \frac{p\left(\boldsymbol{e}^{*}, \boldsymbol{z}^{*} \mid \alpha, \beta, \boldsymbol{x}^{*}=a \in \boldsymbol{a}_{s}^{*}, \boldsymbol{e}, \boldsymbol{x}, \boldsymbol{z}, \boldsymbol{\theta}, \boldsymbol{\phi}, \boldsymbol{a}_{\mathcal{S}}\right)}{q\left(\boldsymbol{x}^{*}, \boldsymbol{z}^{*}\right)} .
\end{aligned}
$$

Referring to the derivation of the ASTM parameters in Sect. 4.3, we can finally obtain the update for the $\gamma_{s s_{s i} a t}^{*}$.

$$
\gamma_{s s_{s i} a t}^{*} \propto \frac{\left(\alpha+N_{a t}^{* / s i}+N_{a t}\right)\left(\beta+N_{t m}^{* / s i}+N_{t m}\right)}{\left(M \beta+N_{t}^{* / s i}+N_{t}\right)} .
$$

In expectation of the $\gamma_{s e_{s i} a t}^{*}$ of the maximum likelihood, this update is repeated until a convergence condition is satisfied.

\section{Experiment}

\subsection{Experimental Conditions}

We evaluated the acoustic scene estimation performance for the proposed and conventional models with two kinds of sound data sets, both of which were recorded in several reallife situations. As one data set, we recorded 11,105 sounds in a living room $\left(22.8 \mathrm{~m}^{2}\right.$, reverberation time: $0.31 \mathrm{sec}$.), which included 9 categories of user activities: "chatting," "cooking," "eating dinner," "operating PC," "reading a newspaper," "vacuuming," "walking," "washing dishes," and "watching TV." We separated them into 9,802 sounds for parameter training and 1,303 sounds for evaluation. As 
Table 2 Experimental conditions

\begin{tabular}{ll}
\hline Sampling rate/quantization & $16 \mathrm{kHz} / 16$ bits \\
Frame size/shift & $512 / 256$ \\
Acoustic event class size & $32-512$ \\
Acoustic topic class size & $20,50,100$ \\
Acoustic event sequence size & $16 \mathrm{~s}, 1,000$ frames \\
Hyperparameter $\alpha / \beta$ & $3.3333 / 0.1$ \\
Iteration number of CVB0 & 1,000 \\
\hline
\end{tabular}

the other data set, we recorded 25,277 sounds which included 8 categories of situations: "bicycle," "bus," "car," "meeting," "office," "park," "street," and "train.” We separated them into 21,461 sounds for parameter training and 3,816 sounds for evaluation. In the acoustic event recognition stage, 12-dimensional MFCC features were calculated from every segmented acoustic signal with $50 \%$ overlap, and acoustic event models were learned by using GC with 32512 acoustic event classes. Each $\boldsymbol{e}_{s}$ was calculated from a 16-s signal that included 1,000 acoustic events. The experimental conditions for the acoustic scene estimation are listed in Table 2.

\subsection{Generalization Performance}

We evaluated the generalization performance of the ASTM, ASM, and ATM using perplexity. Perplexity evaluates how well a model predicts a data set or acoustic scenes, and a lower perplexity indicates a better generalization performance. In particular, the perplexity for the ASTM was calculated as follows.

$$
\begin{aligned}
& \operatorname{Perplexity}\left(\boldsymbol{e}_{s} \mid \boldsymbol{a}_{s}\right)=\exp \left[-\frac{1}{N_{\boldsymbol{e}_{s}}} \log p\left(\boldsymbol{e}_{s} \mid \boldsymbol{a}_{\mathcal{S}}\right)\right] \\
& =\exp \left[-\frac{1}{N_{\boldsymbol{e}_{S}}} \log \sum_{\boldsymbol{x}} \sum_{z} \iint p\left(\boldsymbol{x} \mid \boldsymbol{a}_{\mathcal{S}}\right) p(\boldsymbol{\theta} \mid \alpha) p(\boldsymbol{z} \mid \boldsymbol{\theta}, \boldsymbol{x}) p(\boldsymbol{\phi} \mid \beta) p(\boldsymbol{e} \mid \boldsymbol{\phi}, \boldsymbol{z}) d \boldsymbol{\theta} d \boldsymbol{\phi}\right] .
\end{aligned}
$$

For perplexity with the ASM and ATM, we calculated it in a manner similar to [23]. For the perplexities with the ASTM and ATM, we chose $T=20,50,100$ as the number of acoustic topics, and for all models, we used the same test set including the above nine categories of user activities and eight categories of situations. As shown in Fig. 6, the ASTM decreased the perplexity more compared with the ASM and ATM in all number of classes of acoustic events; thus, the ASTM achieves better generalization performance in modeling acoustic scenes and sub-topics with the consequent $\boldsymbol{e}_{s}$ since it can flexibly model acoustic scenes that have subordinate categories included with the acoustic scene information and the latent structure of acoustic event sequences.

\subsection{Acoustic Scene Estimation Accuracy}

The averaged acoustic scene estimation accuracies (Fmeasure) of the nine categories of user activities and eight categories of situations are shown in Figs. 7 and 8. Here, the F-measure is calculated by using the harmonic mean of the precision and recall as follows.

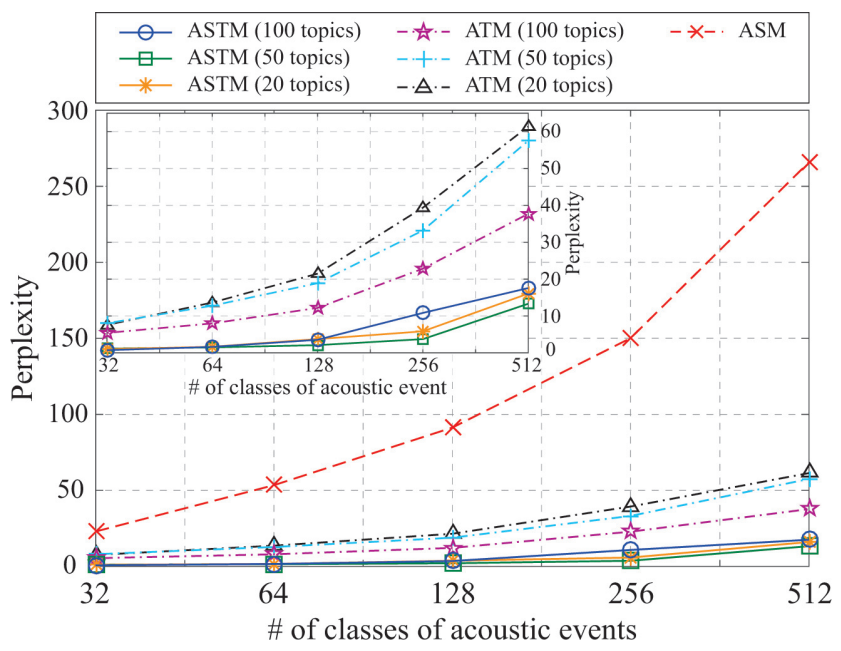

Fig. 6 Averaged perplexities for proposed and conventional models

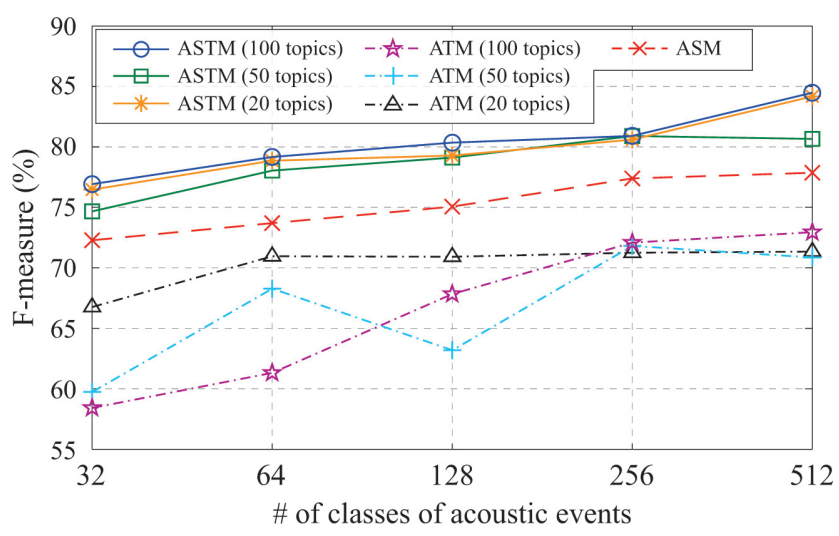

Fig. 7 Averaged accuracy (F-measure) in nine categories of user activity with ASTM, ATM, and ASM

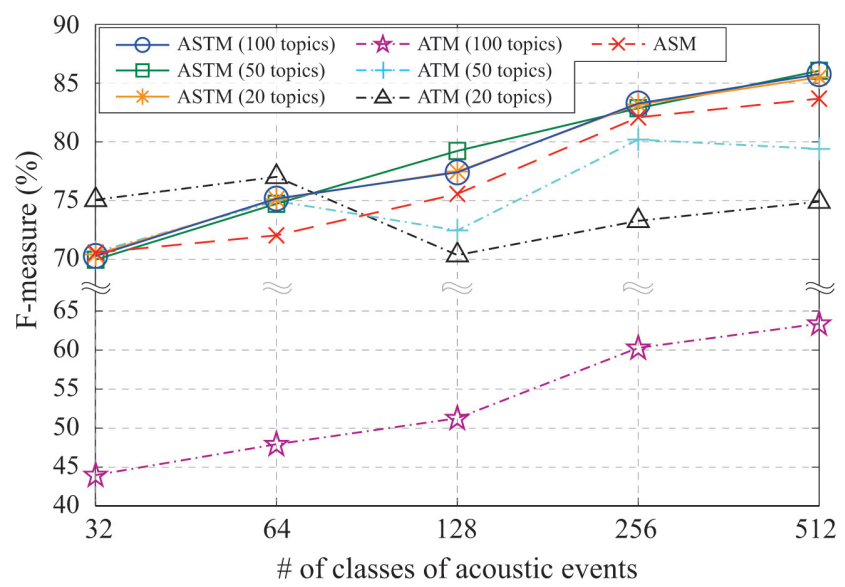

Fig. 8 Averaged accuracy (F-measure) in eight categories of situations with ASTM, ATM, and ASM

$$
\text { F-measure }=2 \cdot \frac{\text { Precision } \cdot \text { Recall }}{\text { Precision }+ \text { Recall }} .
$$

In Fig. 7, the estimation accuracy for the ASTM increased about three points compared with the ASM. For the best 


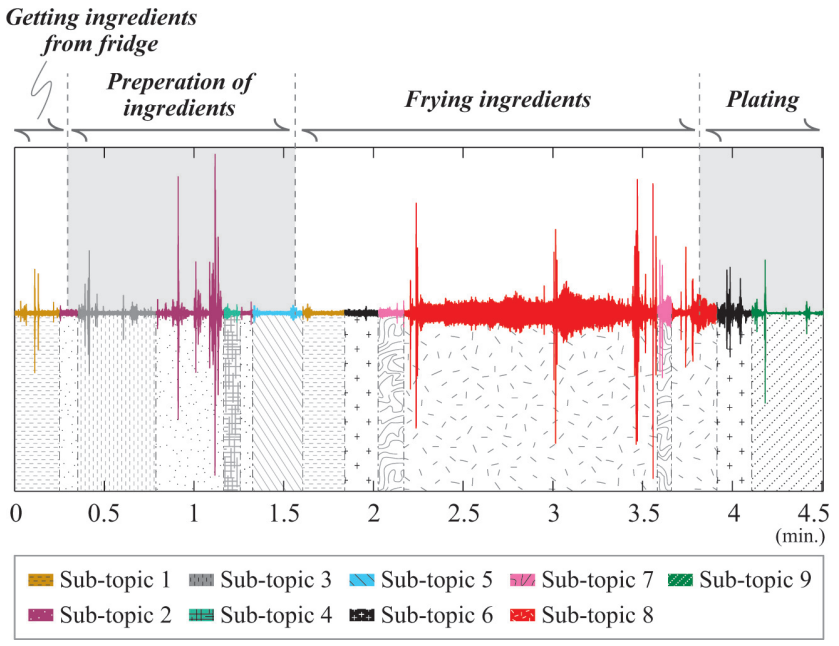

Fig. 9 Correspondence between subordinate categories of user activity "cooking" and estimated acoustic sub-topics with proposed model (ASTM)

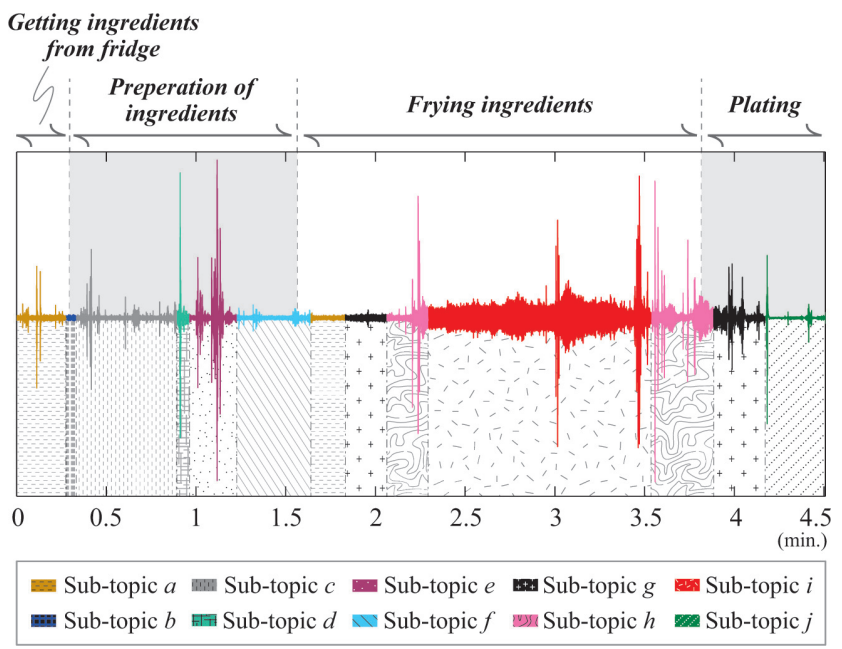

Fig. 10 Correspondence between subordinate categories of user activity "cooking" and estimated acoustic sub-topics with conventional model (ATM)

results in the nine categories of user activities $(T=100$, $M=512$ ), the ASTM correctly classified $84.5 \%$ of the test data set, while the ASM $(M=512)$ and ATM $(T=100$, $M=512$ ) correctly classified $77.9 \%$ and $73.0 \%$. For the eight categories of acoustic scenes, the estimation accuracy for the ASTM increased about two points with the ASM, and for the best results, the ASTM $(T=100, M=512)$ correctly classified $85.8 \%$ of the test data set, while the ASM $(M=512)$ and ATM $(T=50, M=256)$ correctly classified $83.7 \%$ and $80.2 \%$. These results suggest that some acoustic scenes had more than one subordinate category of acoustic scenes; therefore, the ASTM offers advantages in modeling and estimating those kinds of acoustic scenes compared with the ASM.

\subsection{Acoustic Sub-Topic Estimation}

For detailed investigation, we compared sub-topic estima-

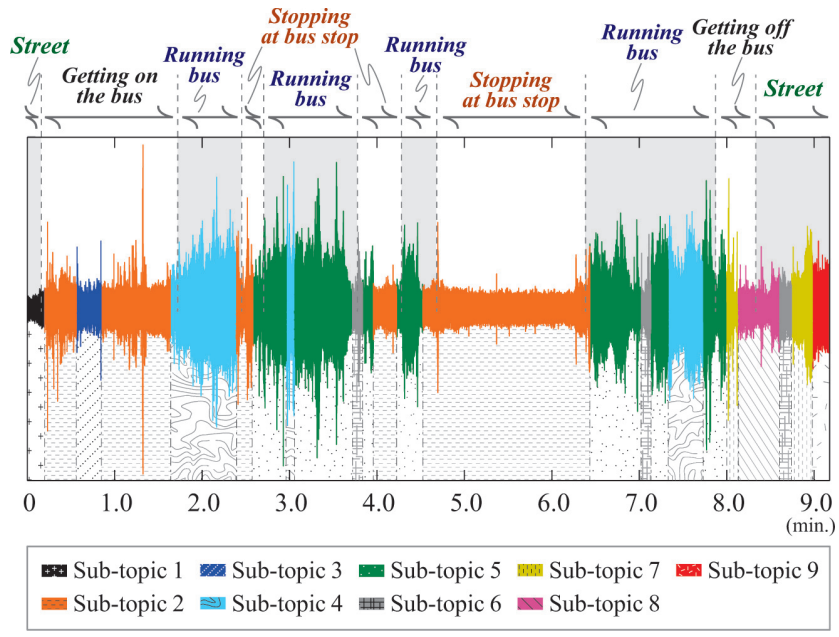

Fig. 11 Correspondence between subordinate categories of situation "bus" and estimated acoustic sub-topics with proposed model (ASTM)

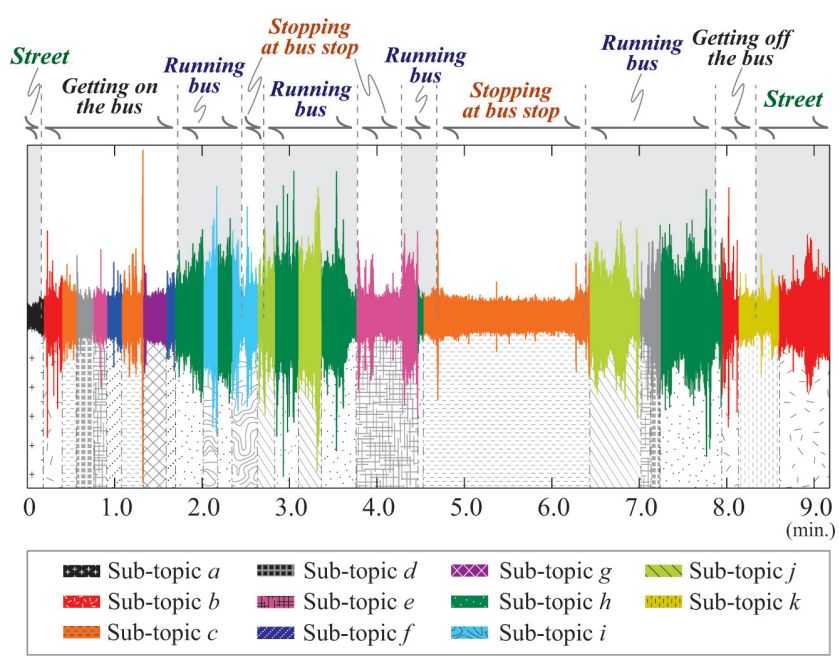

Fig. 12 Correspondence between subordinate categories of situation "bus" and estimated acoustic sub-topics with conventional model (ATM)

tion results in some acoustic scenes with actual subordinate categories of their acoustic scenes. Figures 9 and 10 show the acoustic sub-topic estimation results of "cooking" with a test sound with the ASTM and ATM, respectively, and Figs. 11 and 12 indicate the estimation results for "bus" when we set $T=50, M=512$. Here, there is no acoustic topic variables for the ASM, and therefore, we cannot analyze acoustic topics with the ASM. The subordinate categories of acoustic scenes described in the upper part of the figures indicate actual manually labeled categories, and the color-coded acoustic signals denote the correspondence relationships between acoustic event sequences and acoustic sub-topics estimated by using the ASTM and ATM. In the case of the estimation result of "cooking," for both models, the estimated acoustic sub-topics agreed well with the actual subordinate categories of acoustic scenes, and these results show that the ASTM and ATM can generally extract subordinate categories of acoustic scenes. For instance, in Fig. 9, 
"preparation of ingredients" and "frying ingredients" agreed well with sub-topics 2,3 , and 8 . However, in the case of the estimation result for "bus," the ASTM extracted subordinate categories of acoustic scenes through less acoustic subtopics more precisely than did the ATM. In Fig. 11, "running bus" and "stopping at bus stop" agreed well with sub-topics 4, 5, and 2, whereas in Fig. 12, they are sometimes confused. This result suggests that the proposed method can extract not only acoustic scenes but also their subordinate categories more precisely.

\section{Conclusion and Future Work}

In this paper, we proposed an acoustic scene estimation method based on a hierarchical generative model of acoustic event sequences that is associated with acoustic scenes and their sub-topics, in which each acoustic scene is represented by a probability distribution over acoustic sub-topics, and each acoustic sub-topic is represented as the probability distribution over acoustic events. We also proposed a parameter estimation method for the proposed model based on CVBO and an acoustic scene estimation method that has a three-step process: recognizing frame-by-frame acoustic events to generate an acoustic event sequence, estimating acoustic scene model parameters, and predicting acoustic scenes in unlabeled acoustic signals with estimated model parameters.

Acoustic scene estimation experiments with real-life sounds showed that the proposed method improved perplexity; that is, the proposed method achieved better generalization performance in modeling acoustic scenes that have more than one subordinate category of acoustic scenes. Experiments also showed that the proposed method improved the acoustic scene estimation accuracy an average of more than 2-3 points compared with the existing acoustic scene estimation method, and the proposed method also enables the extraction of subordinate categories of acoustic scenes more correctly.

As future work, we need to quantitatively evaluate how precisely the proposed method extracts the subordinate categories of acoustic scenes through acoustic sub-topics. In addition, we will extend the proposed method to an online method that can apply to sequentially obtained sounds and can analyze acoustic scenes in real time.

\section{References}

[1] Y.-T. Peng, C.-Y. Lin, M.-T. Sun, and K.-C. Tsai, "Healthcare audio event classification using hidden Markov models and hierarchical hidden Markov models," Proc. IEEE International Conference on Multimedia and Expo (ICME), pp.1218-1221, 2009.

[2] A. Harma, M.F. McKinney, and J. Skowronek, "Automatic surveillance of the acoustic activity in our living environment," Proc. IEEE International Conference on Multimedia and Expo (ICME), pp.634-637, 2005.

[3] A.J. Eronen, V.T. Peltonen, J.T. Tuomi, A.P. Klapuri, S. Fagerlund, T. Sorsa, G. Lorho, and J. Huopaniemi, "Audio-based context recognition," IEEE Trans. Audio, Speech, Language Process., vol.14, no.1, pp.321-329, 2006.
[4] K. Imoto and N. Ono, "Spatial-feature-based acoustic scene analysis using distributed microphone array," Proc. European Signal Processing Conference (EUSIPCO), pp.739-743, 2015.

[5] Y. Ohishi, D. Mochihashi, T. Matsui, M. Nakano, H. Kameoka, T. Izumitani, and K. Kashino, "Bayesian semi-supervised audio event transcription based on Markov Indian buffet process," Proc. IEEE International Conference on Acoustics, Speech and Signal Process. (ICASSP), pp.3163-3167, 2013.

[6] A. Temko, R. Malkin, C. Zieger, D. Macho, C. Nadeu, and M. Omologo, "CLEAR evaluation of acoustic event detection and classification systems," Springer Berlin Hidelberg, pp.311-322, 2007.

[7] A. Mesaros, T. Heittola, A. Eronen, and T. Virtanen, "Acoustic event detection in real life recordings," Proc. 18th European Signal Processing Conference (EUSIPCO), pp.1267-1271, 2010.

[8] D. Barchiesi, D. Giannoulis, D. Stowell, and M.D. Plumbley, "Acoustic scene classification: Classifying environments from the sounds they produce," IEEE Signal Process. Mag., vol.32, no.3, pp.16-34, 2015.

[9] M.A.M. Ahaikh, M.K.I. Molla, and K. Hirose, "Automatic life-logging: A novel approach to sense real-world activities by environmental sound cues and common sense," Proc. 11th International Conference on Computer and Information Technology (ICCIT), pp.294-299, 2008.

[10] T. Heittola, A. Mesaros, A. Eronen, and A. Klapuri, "Audio content recognition using audio event histograms," Proc. 18th European Signal Processing Conference (EUSIPCO), pp.1272-1276, 2010.

[11] S. Kim, S. Narayanan, and S. Sundaram, "Acoustic topic models for audio information retrieval," Proc. 2009 IEEE Workshop on Applications of Signal Processing to Audio and Acoustics (WASPAA), pp.37-40, 2009.

[12] K. Lee and D.P.W. Ellis, "Audio-based semantic concept classification for consumer video," IEEE Trans. Audio, Speech, Language Process., vol.18, no.6, pp.1406-1416, 2010.

[13] K. Imoto, Y. Ohishi, H. Uematsu, and H. Ohmuro, "Acoustic scene analysis based on latent acoustic topic and event allocation," Proc. IEEE International Workshop on Machine Learning for Signal Processing (MLSP), pp.1-6, 2013.

[14] K. Imoto and N. Ono, "Acoustic scene analysis from acoustic event sequence with intermittent missing event," Proc. IEEE International Conference on Acoustics, Speech and Signal Processing (ICASSP), pp.156-160, 2015.

[15] C.M. Bishop, Pattern Recognition and Machine Learning, Springer, 2006.

[16] T. Joachims, "Learning to classify text using support vector machines: Methods, theory, and algorithms," J. Comput. Linguist., vol.29, pp.655-664, 2003.

[17] A.P. Dempster, N.M. Laird, and D.B. Rubin, "Maximum likelihood from incomplete data via the em algorithm," J. Royal Statistical Society, vol.39, no.1, pp.1-38, 1977.

[18] A. Asuncion, M. Welling, P. Smyth, and Y.W. Teh, "On smoothing and inference for topic models," Proc. 25th Conference on Uncertainty in Artificial Intelligence (UAI), pp.27-34, 2009.

[19] H. Attias, "A variational Bayesian framework for graphical models," In Adv. Neural Inf. Proc. Syst. 12, pp.209-215, 2000.

[20] R.M. Neal, "Probabilistic inference using Markov chain Monte Carlo methods," Dept. of Comput. Sci., Univ. of Toronto, Tech. Rep. CRG-TR-93-1, 1993.

[21] T.L. Griffiths and M. Steyvers, "Finding scientific topics," PNAS, vol.1, pp.5228-5235, 2004.

[22] Y.W. Teh, D. Newman, and M. Welling, "A collapsed variational Bayesian inference algorithm for latent dirichlet allocation," In Adv. Neural Inf. Proc. Syst. (NIPS), pp.1353-1360, 2006.

[23] M. Rosen-Zvi, T.L. Griffiths, M. Steyvers, and P. Smyth, "The author-topic model for authors and documents," Proc. 20th Conference on Uncertainty in Artificial Intelligence, pp.487-494, 2004. 


\section{Appendix: Detailed Discussion of ASTM Parameter Estimation}

In this appendix, we discuss the derivation of the ASTM parameters in detail.

\section{A.1 Derivation of Eq. (6)}

The first equation of Eq. (6) can be rewritten as follows.

$$
\begin{aligned}
& \sum_{\boldsymbol{x}} \sum_{z} q(\boldsymbol{x}, \boldsymbol{z}) \log p\left(\boldsymbol{e}, \boldsymbol{z}, \boldsymbol{x} \mid \alpha, \beta, \boldsymbol{a}_{\mathcal{S}}\right) \\
& =\sum_{\boldsymbol{x}} \sum_{z} q(\boldsymbol{x}, \boldsymbol{z}) \log \iint p\left(\boldsymbol{x} \mid \boldsymbol{a}_{\mathcal{S}}\right) p(\boldsymbol{\theta} \mid \alpha) p(\boldsymbol{z} \mid \boldsymbol{\theta}, \boldsymbol{x}) p(\boldsymbol{\phi} \mid \beta) p(\boldsymbol{e} \mid \boldsymbol{\phi}, \boldsymbol{z}) d \boldsymbol{\theta} d \boldsymbol{\phi} \\
& =\sum_{\boldsymbol{x}} \sum_{z} \prod_{s} \prod_{i} q\left(x_{s i}, z_{s i}\right) \cdot \log \iint \prod_{s} \prod_{i} \frac{1}{A_{s}} \\
& \cdot \prod_{a}\left\{\frac{\Gamma(T \alpha)}{\Gamma(\alpha)^{T}} \prod_{t} \theta_{a t}^{\alpha-1} \prod_{t} \theta_{a t}^{n_{a t}}\right\} \prod_{t}\left\{\frac{\Gamma(M \beta)}{\Gamma(\beta)^{M}} \prod_{m} \phi_{t m}^{\beta-1} \prod_{m} \phi_{t m}^{n_{m, t}}\right\} d \boldsymbol{\theta} d \boldsymbol{\phi} .
\end{aligned}
$$

Now, we consider an integral of the Dirichlet distribution as follows.

$$
\begin{aligned}
\int \operatorname{Dir}(\mu \mid \zeta) d \mu & =\int \frac{\Gamma\left(\sum_{j} \zeta_{j}\right)}{\prod_{j} \Gamma\left(\zeta_{j}\right)} \prod_{j} \mu_{j}^{\zeta_{j}-1} d \mu=1 \\
\int \prod_{j} \mu_{j}^{\zeta_{j}-1} d \mu & =\frac{\prod_{j} \Gamma\left(\zeta_{j}\right)}{\Gamma\left(\sum_{j} \zeta_{j}\right)} .
\end{aligned}
$$

Substituting Eq. (A.2) into Eq. (A.1), Eq. (6) can be calculated as follows.

$$
\begin{aligned}
& \sum_{x} \sum_{z} q(\boldsymbol{x}, \boldsymbol{z}) \log p\left(\boldsymbol{e}, \boldsymbol{z}, \boldsymbol{x} \mid \alpha, \beta, \boldsymbol{a}_{\mathcal{S}}\right) \\
& \cdot \prod_{a}\left\{\frac{\Gamma(T \alpha)}{\Gamma(\alpha)^{T}} \cdot \frac{\prod_{t} \Gamma\left(\alpha+n_{. . a t}\right)}{\Gamma\left(T \alpha+n_{. . a .}\right)}\right\} \prod_{t}\left\{\frac{\Gamma(M \beta)}{\Gamma(\beta)^{M}} \cdot \frac{\prod_{m} \Gamma\left(\beta+n_{. m . t}\right)}{\Gamma\left(M \beta+n_{. . t}\right)}\right\} \\
& \cdot \prod_{a}\left\{\frac{\Gamma(T \alpha)}{\Gamma\left(T \alpha+n_{. . . .}\right)} \prod_{t} \frac{\Gamma\left(\alpha+n_{. a t}\right)}{\Gamma(\alpha)}\right\} \prod_{t}\left\{\frac{\Gamma(M \beta)}{\Gamma\left(M \beta+n_{. . t}\right)} \prod_{m} \frac{\Gamma\left(\beta+n_{. m . t}\right)}{\Gamma(\beta)}\right\} .
\end{aligned}
$$

\section{A.2 Derivation of Eq. (8)}

Then, we discuss the derivation of Eq. (8) in detail. We first consider the following joint probability.

$$
\begin{aligned}
& \frac{p\left(\boldsymbol{e}, \boldsymbol{x}^{\prime s i}, x_{s i}=a, \boldsymbol{z}^{/ s i}, z_{s i}=t \mid \alpha, \beta, \boldsymbol{a}_{\mathcal{S}}\right)}{p\left(\boldsymbol{e}^{s i s i}, \boldsymbol{x}^{s i}, \boldsymbol{z}^{\mid s i} \mid \alpha, \beta, \boldsymbol{a}_{\mathcal{S}}\right)}
\end{aligned}
$$

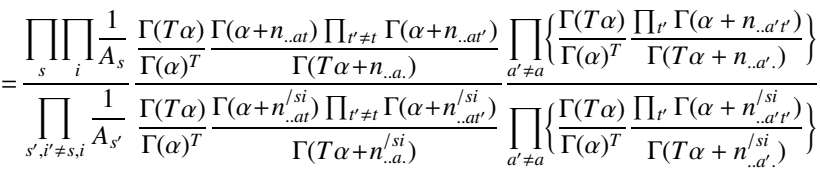

$$
\begin{aligned}
& \frac{\Gamma(M \beta)}{\Gamma(\beta)^{M}} \frac{\Gamma\left(\beta+n_{m . t . t}\right) \prod_{m^{\prime} \neq m} \Gamma\left(\beta+n_{m^{\prime} . t}\right)}{\Gamma\left(M \beta+n_{. t .}\right)} \prod_{t^{\prime} \neq t}\left\{\frac{\Gamma(M \beta)}{\Gamma(\beta)^{M}} \frac{\prod_{m^{\prime}} \Gamma\left(\beta+n_{. m^{\prime}, t^{\prime}}\right.}{\Gamma\left(M \beta+n_{. . . t^{\prime}}\right)}\right\}
\end{aligned}
$$

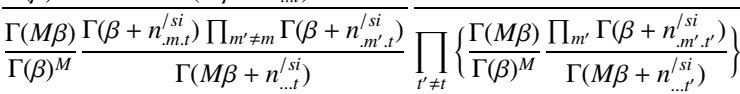

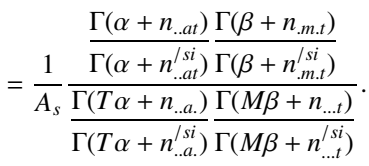

Here, introducing $\Gamma(x+1) / \Gamma(x)=x$ into Eq. (A.4), it can be described as follows.

$$
\frac{p\left(\boldsymbol{e}, \boldsymbol{x}^{/ s i}, x_{s i}=a, \boldsymbol{z}^{/ s i}, z_{s i}=t \mid \alpha, \beta, \boldsymbol{a}_{\mathcal{S}}\right)}{p\left(\boldsymbol{e}^{/ s i}, \boldsymbol{x}^{/ s i}, \boldsymbol{z}^{/ s i} \mid \alpha, \beta, \boldsymbol{a}_{\mathcal{S}}\right)}=\frac{1}{A_{s}} \frac{\alpha+n_{. . a t}^{/ s i}}{T \alpha+n_{. . a .}^{/ s i}} \frac{\beta+n_{. m . t}^{/ s i}}{M \beta+n_{\ldots . t}^{/ s i}} .
$$

Substituting Eq.(A.5) into Eq.(8), the last equation of Eq. (8) can be derived.

\section{A.3 Derivation of the update for ASTM parameters}

Finally, we derive the update of Eq. (10). Substituting Eqs. (8) and (9) into $\partial \mathcal{F}\left[q\left(x_{s i}, z_{s i}\right)\right] / \partial q\left(x_{s i}, z_{s i}\right)=0$, we can obtain the following equation.

$$
\begin{aligned}
& \frac{\partial}{\partial \gamma_{s e_{s i} a t}} \sum_{\boldsymbol{x}} \sum_{z} q(\boldsymbol{x}, \boldsymbol{z}) \log p\left(\boldsymbol{e}, \boldsymbol{x}, z \mid \alpha, \beta, \boldsymbol{a}_{\mathcal{S}}\right) \\
& -\frac{\partial}{\partial \gamma_{s e_{s i} a t}} \sum_{x} \sum_{z} q(\boldsymbol{x}, \boldsymbol{z}) \log q(\boldsymbol{x}, \boldsymbol{z})=0
\end{aligned}
$$

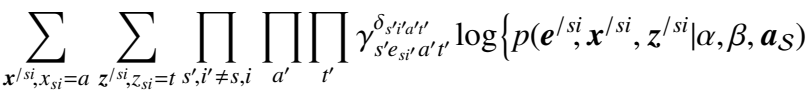

$$
\begin{aligned}
& \left.\cdot \frac{\alpha+n_{. . a^{\prime} t^{\prime}}^{/ s i}}{T \alpha+n_{. . a^{\prime} .}^{/ s i}} \cdot \frac{\beta+n_{. e_{s^{\prime} t^{\prime}, t^{\prime}}}^{/ s i}}{M \beta+n_{. . . t^{\prime}}^{/ s i}}\right\}-\log \gamma_{s e_{s i a t} a t}+\text { const. }=0
\end{aligned}
$$

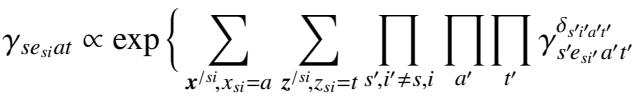

$$
\begin{aligned}
& \cdot \log \left\{p\left(\boldsymbol{e}^{/ s i}, \boldsymbol{x}^{/ s i}, \boldsymbol{z}^{/ s i} \mid \alpha, \beta, \boldsymbol{a}_{\mathcal{S}}\right) \cdot \frac{\alpha+n_{. a^{\prime} t^{\prime}}^{/ s i}}{T \alpha+n_{. . a^{\prime} .}^{/ s i}} \cdot \frac{\beta+n_{. s_{s^{\prime} t^{\prime}, t^{\prime}}}^{/ s i}}{M \beta+n_{. . t^{\prime}}^{/ s i}}\right\} .
\end{aligned}
$$

Considering that $p\left(\boldsymbol{e}^{/ s i}, \boldsymbol{x}^{/ s i}, \boldsymbol{z}^{/ s i} \mid \alpha, \beta, \boldsymbol{a}_{\mathcal{S}}\right)$ does not depend on $x_{s i}$ and $z_{s i}$, Eq. (A.4) can be rewritten as follows.

$$
\begin{aligned}
& \gamma_{s e_{s i a t} a t} \propto \exp \left[\sum_{x^{s i,}, x_{s i}=a} \sum_{z^{\prime s i}, z_{s i}=t} \prod_{s^{\prime}, i^{\prime} \neq s, i} \prod_{a^{\prime}} \prod_{t^{\prime}} \gamma_{s^{\prime} e_{s^{\prime}}}^{\delta_{s^{\prime} a^{\prime}} a^{\prime} t^{\prime}}\right. \\
& \left.\left\{\log \left(\alpha+n_{. . a^{\prime} t^{\prime}}^{/ s i}\right)+\log \left(\beta+n_{. e_{s^{\prime} t^{\prime}, t^{\prime}}^{\prime}}^{/ s i}\right)-\log \left(M \beta+n_{. . . t^{\prime}}^{/ s i}\right)\right\}\right] .
\end{aligned}
$$

By introducing the form of expectation of $q\left(x^{/ s i}, z^{/ s i}\right)$, we can derive Eq. (10) from Eq. (A.7). 


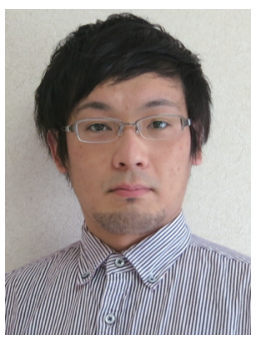

Keisuke Imoto received B.E., M.E. degrees from the Kyoto University in 2008 and 2010. He joined the Nippon Telegraph and Telephone Corporation (NTT) in 2010. He is now a Ph.D. student at SOKENDAI (The Graduate University for Advanced Studies), and he has been engaged in research on acoustic event detection and microphone array signal processing. He is a member of IEEE, IPSJ, and ASJ.

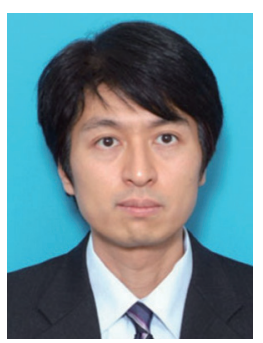

Suehiro Shimauchi received B.E., M.E., and $\mathrm{Ph} . \mathrm{D}$. degrees from the Tokyo Institute of Technology in 1991, 1993, and 2007, respectively. Since joining the Nippon Telegraph and Telephone Corporation (NTT) in 1993, he has been engaged in research on acoustic signal processing. He is now a senior research engineer at NTT Media Intelligence Laboratories. He is a senior member of IEEE and a member of ASJ. 